\title{
Fisioterapia baseada no treinamento de dupla tarefa no equilíbrio de indivíduos com Doença de Parkinson
}

\author{
Isabela Andrelino de Almeida', Maria Eduarda Brandão Bueno ${ }^{1}$, Ana Carolina dos Reis Andrello², \\ Cyntia Letícia Batistetti', Luana Beatriz Lemes ${ }^{1}$, Natália Mariano Barboza', \\ Lucio Baena de Melo ${ }^{3}$, Suhaila Mahmoud Smaili Santos ${ }^{4}$
}

\begin{abstract}
RESUMO
Objetivo: Investigar a efetividade de um protocolo de tratamento fisioterápico baseado no treino de duplas tarefas no equilíbrio de indivíduos com doença de Parkinson. Métodos: Nove indivíduos foram avaliados antes e após um programa fisioterápico baseado em um protocolo de exercícios de dupla tarefa, com terapia de 60 minutos, 2x/semana. Os seguintes instrumentos foram utilizados para avaliação: Escala Unificada para Avaliação da doença de Parkinson (UPDRS), Balance Evaluation Systems Test (BESTest) e Protocolo de avaliação do Balance. Resultados: Foram encontradas diferença significantes nos valores do domínio motor e na pontuação total da UPDRS, nos valores referentes ao BESTest em duas seções e escore total, além de duas posturas do Protocolo de avaliação do Balance. Conclusão: A intervenção foi eficaz no desfecho equilíbrio, escores motor e escore total da UPDRS, o que sugere ser um tratamento adequado sobre o equilíbrio e evolução da doença no grupo estudado.
\end{abstract}

Descritores: Doença de Parkinson; Equilíbrio Postural; Modalidades de Fisioterapia; Reabilitação.

\section{Physical therapy treatment based in dual task training in balance to individuals with parkinson's disease}

\begin{abstract}
Objective: This study was designed to investigate the effectiveness of a physical therapy treatment protocol based in dual task training in balance in individuals with Parkinson's disease. Méthods: Nine patients were evaluated before and after a physical therapy program based on an exercise protocol dual task, with 60 minutes, 2x/week. The following variables were investigated: Unified Parkinson's Disease Rating Scale (UPDRS), Balance Evaluation Systems Test (BESTest) and measurements of the Balance Protocol. Results: Significant differences were found in the motor domain values and the total score of UPDRS, in two sections and the total score values of BESTest, and two postures of Balance Assessment Protocol. Conclusion: The intervention was effective in balance, motor scores and total scores of the UPDRS, suggesting be an appropriate treatment for balance and evolution of the disease in the studied group.
\end{abstract}

Descriptors: Parkinson Disease; Postural Balance; Physical Therapy Modalities; Rehabilitation.

\footnotetext{
${ }^{1}$ Fisioterapeuta e Residente em Fisioterapia Neurofuncional Adulto pela Universidade Estadual de Londrina (UEL) Londrina, PR, Brasil.

${ }^{2}$ Fisioterapeuta pela Universidade Estadual de Londrina (UEL) Londrina, PR, Brasil.

${ }^{3}$ Médico Neurologista pela Universidade Estadual de Londrina (UEL) Londrina, PR, Brasil.

${ }^{4}$ Doutora em Fisiopatologia em Clínica Médica pela Universidade Estadual Paulista (UNESP), Botucatu, SP, Brasil.
} 


\section{Introdução}

O controle postural é a capacidade em manter a projeção do centro de massa do corpo dentro dos limites da base de apoio sob condições estáticas e dinâmicas, e envolve o controle da posição do corpo no espaço com o objetivo de assegurar estabilidade e orientação ${ }^{1}$. Depende dos sistemas visual, vestibular, somatossensorial, nervoso, biomecânico e cognitivo, que são expostos a diferentes tipos de disfunções².

Idosos são mais suscetíveis a adquirir distúrbios do equilíbrio devido algumas deficiências, como perda multissensorial, fraqueza muscular e limitações ortopédicas e cognitivas culminando em perdas funcionais, impacto sobre a qualidade de vida e elevado custo ao sistema de saúde ${ }^{3}$. Adicionalmente, várias doenças neurológicas estão associadas à instabilidade postural, como a doença de Parkinson (DP), sendo o distúrbio do movimento mais comum que acomete o sistema nervoso central e de maior incidência em pessoas idosas afetando entre 1 e $3 \%$ desta população $0^{4,5}$. Clinicamente a doença é marcada pela presença de tremor de repouso, rigidez, bradicinesia, instabilidade postural e alterações na marcha ${ }^{6,7}$.

As alterações posturais somadas às outras alterações levam a instabilidade postural na DP. Acredita-se que a deterioração do equilíbrio, implícita na instabilidade postural, possa estar relacionada à perda da habilidade em controlar os movimentos intencionais do centro de massa corporal sobre a base de suporte durante a realização de atividades que envolvem transferências de peso ${ }^{8}$. Além disso, a dificuldade na aquisição da estabilidade postural na DP referese a alteração na ordenação do movimento, em sua origem subcortical e não no músculo propriamente dito ${ }^{9}$. Nessa linha, estudos sinalizam a necessidade de avaliar como as alterações provocadas pela DP interferem no equilíbrio e na funcionalidade desses pacientes, no intuito de selecionar a abordagem mais adequada para a reabilitação ${ }^{10}$.

Quando se realiza duas tarefas motoras ou uma tarefa motora e uma cognitiva simultaneamente podemos classificá-las como atividades de duplas tarefas e em muitas atividades diárias é altamente vantajoso executar mais de uma tarefa ao mesmo tempo. Em circunstâncias normais, quando há realização concomitante de tarefas motoras e cognitivas, as atividades motoras são desempenhadas de forma automática, pois não requerem recursos atencionais conscientes. Entretanto, quando se apresenta alteração cognitiva e/ou no controle motor, naturalmente há uma demanda atencional maior, podendo comprometer o desempenho de uma ou de ambas ${ }^{11,12}$. O equilíbrio também pode ser considerado um dupla tarefa, pois apesar do equilíbrio parecer uma tarefa única, é necessário a integração dos sistemas sensorial, nervoso e músculo-esquelético para que o centro de massa corporal mantenha-se dentro de uma base de suporte entre os limites de estabilidade ${ }^{12}$.

Estudos sugerem que intervenções positivas para os indivíduos com DP sobre a melhora da marcha, mobilidade, equilíbrio, agilidade e nos escores da UPDRS devem englobar abordagens baseadas no conceito Bobath, treino de equilíbrio, treino de marcha com pistas visuais, sensoriais e auditivas, facilitação neuromuscular, entre outras ${ }^{13,14,15}$.

Está bem estabelecido que o treinamento baseado nas duplas tarefas pode ser usado como estratégia para estimular a melhora da marcha, e não se sabe ao certo o seu impacto no equilíbrio desses pacientes ${ }^{16,17}$, deste modo o objetivo do presente estudo foi verificar a efetividade do treinamento da dupla tarefa na progressão da doença e no equilibrio em indivíduos com doença de Parkinson.

\section{Metodologia}

Trata-se de uma série de casos prospectiva composta por um grupo de nove indivíduos, com diagnóstico médico de DP, caracterizado por amostra de conveniência, provenientes do Ambulatório Médico do Hospital de Clínicas da Universidade Estadual de Londrina.

Foram incluídos no estudo indivíduos com faixa etária acima de 65 anos, não institucionalizados, classificados entre os estágios 1,5 e 3 na escala de Hoehn e Yahr, que aceitassem participar da pesquisa, após assinatura do termo de consentimento livre e esclarecido, de acordo com os critérios do Comitê Local de Ética em Pesquisa com Seres Humanos, sob parecer de aprovação CEP-UEL n 066/2011, e também segundo as normas da Resolução do Conselho Nacional de Saúde n 466/12.

Foram excluídos os indivíduos que apresentavam outras formas de Parkinsonismo; outras patologias neurológicas associadas como doenças vasculares, traumáticas ou desmielinizantes; distúrbios vestibulares, como labirintite, vertigem posicional paroxística benigna e zumbido; distúrbios cardiovasculares, como angina instável; distúrbios musculoesqueléticos, que limitem a amplitude de movimento; distúrbios cognitivos ou de compreensão, como demências; distúrbios visuais ou auditivos que afetassem o desempenho motor; indivíduos que deambulassem com órteses ou dispositivos de auxílio à marcha e que realizassem outro tratamento terapêutico além do medicamentoso. Aqueles que tiveram alteração na medicação durante o estudo ou que totalizaram mais de 3 faltas nas terapias foram considerados como perda. 


\section{Procedimentos de avaliação}

Os pacientes foram avaliados sempre no estágio "on" da medicação (momento em que o indivíduo está sob melhor efeito do medicamento) e por um mesmo avaliador. Para a avaliação dos participantes foram utilizados os seguintes testes e instrumentos:

- Escala de Estágios de Incapacidade de Hoehn e Yahr Modificada (HY - Degree of Disability Scale): amplamente utilizada para avaliar o estadiamento da doença, de forma rápida e prática que indica o estado geral do paciente. Em seu formato original, compreende cinco estágios de classificação para avaliar a severidade da DP baseandose na instabilidade postural, rigidez, tremor e bradicinesia ${ }^{18}$.

- Mini Exame do Estado Mental (MEEM): utilizada para avaliação das funções cognitivas, composto por questões agrupadas em 7 categorias, cada uma delas desenhada com o objetivo de avaliar funções cognitivas específicas, como orientação para tempo e espaço, registro e lembrança de palavras, linguagem, atenção e cálculo, e capacidade construtiva visual. $O$ escore pode variar de 0 a 30 pontos, e o ponto de corte 23/24 tem boa a excelente sensibilidade e especificidade para 0 diagnóstico de demência ${ }^{19}$.

- Escala Unificada para Avaliação da Doença de Parkinson (Unified Parkinson's Disease Rating Scale -UPDRS): apenas os domínios relacionados ao exame motor e atividades de vida diária. É uma escala amplamente utilizada para monitorar a progressão da doença e avalia sinais, sintomas e determinadas atividades dos indivíduos por meio de autorrelato e observação clínica. É uma escala confiável e válida, sendo composta por 42 itens, divididos em quatro domínios. A pontuação varia de 0 a 4 em cada item, sendo 154 o valor máximo, indicando maior gravidade da doença. No domínio atividade de vida diária a pontuação varia de 0 a 48 pontos e no domínio motor varia de 0 a 52 pontos ${ }^{20}$.

- Protocolo de avaliação do Balance: Optou-se por sua escolha para avaliar o equilíbrio, pois consiste em testes realizados em posturas que requerem alta demanda no dia a dia dos indivíduos. Os indivíduos permanecem de olhos abertos (OA) e fechados (OF), em superfície estável (solo) e sobre superfície instável (espuma) com pés em posição de Romberg (pés unidos), Tandem bilateral (um pé a frente do outro, em posição calcanhar dedos), postura realizada ao caminhar por exemplo, e apoio unipodal bilateral, que ocorre em atividades diárias como subir e descer degraus, e vestir-se por exemplo. 0 tempo de permanência em cada teste é cronometrada em segundos e o objetivo é que 0 avaliado permaneça 30 segundos em cada posição ${ }^{10}$ (Figura 1).

Figura 1 - Protocolo de avaliação do balance
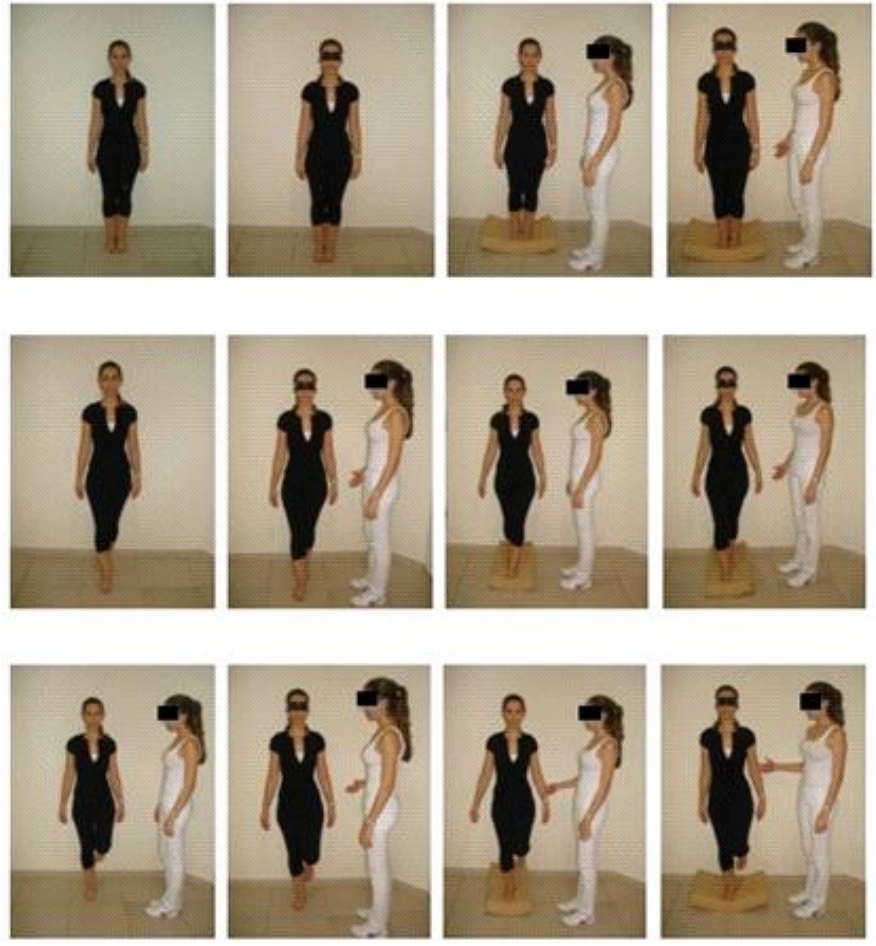
- BESTest (Balance Evaluation Systems Test): O BESTest tem como objetivo avaliar os subsistemas envolvidos no controle do equilíbrio e consiste na execução de 27 tarefas. No estudo de desenvolvimento dessa escala, Horak e colaboradores afirmam que esses subsistemas trabalham em conjunto, mas muitos deles são independentes de acordo com o circuito neural envolvido, de tal maneira que patologias diferentes podem afetar diferentes subsistemas. Indivíduos com DP, por exemplo, podem ter alterações nas reações de passo em resposta a interferências externas, mas possuírem uma orientação sensorial normal que os possibilita permanecer em pé com os olhos fechados em uma superfície instáve $\left.\right|^{10} .0$ teste contém seis seções correspondentes aos subsistemas do equilíbrio: I. Restrições Biomecânicas; II. Limites de estabilidade; III. Transições e ajustes posturais antecipatórios; IV. Respostas posturais à perturbação; V. Orientação sensorial de pé sobre uma base de apoio inclinada e VI. Estabilidade dinâmica da marcha com e sem tarefa cognitiva. Possui um total de 36 itens, cada um pontuado de zero (pior desempenho) a três (melhor desempenho) totalizando 108 pontos. A seção I varia de 0 a 15 pontos, a seção II varia de 0 a 21 pontos, a seção III varia de 0 a 18 pontos, a seção IV varia de 0 a 18 pontos, a seção $V$ varia de 0 a 15 pontos e a seção VI varia de 0 a 21 pontos. Instruções específicas para o examinador e para serem dadas aos pacientes são fornecidas ${ }^{21}$.

\section{Procedimentos de intervenção}

Todos os participantes foram avaliados antes e após a intervenção, sempre por um mesmo avaliador. Para a intervenção foi elaborado um programa de intervenção fisioterapêutica baseado em um protocolo de exercícios de duplas tarefas, desenvolvido de acordo com o conceito Bobath, com terapia de 60 minutos e frequência de duas vezes por semana, durante 12 semanas, totalizando 24 terapias. As terapias foram realizadas em grupo, sendo que cada participante, de acordo com as necessidades individuais, foi acompanhado por um fisioterapeuta previamente treinado. Os materiais utilizados consistiram em bola Bobath, bola dente de leite, cones, caixas coloridas, bambolês, espaguetes, escada horizontal e chapéu chinês. Foram realizados exercícios nas posições sentado e em pé de forma progressiva, evoluindo de tarefas simples para duplas tarefas. Estes exercícios tiveram como objetivos melhorar o padrão de marcha, melhora do equilibrio estático e dinâmico, facilitar as passagens de posturas, melhorar reações automáticas básicas, melhorar a memória, as funções executivas e o tempo da execução das duplas tarefas.

\section{Análise estatística}

A análise estatística foi realizada por meio do programa SPSS 20. Na análise descritiva, os dados foram apresentados conforme a distribuição de normalidade pelo teste de Shapiro-Wilk (média e desvio-padrão ou mediana e quartis). Para as análises de comparação dos momentos pré e pós-intervenção utilizou-se o teste de Wilcoxon. Optou-se pela aplicação dos testes não-paramétricos em função do tamanho amostral reduzido. Foi adotado o valor $\mathrm{P}<0,05$ para que os dados fossem considerados estatisticamente significantes.

\section{Resultados}

A média de idade dos indivíduos foi de $72,77 \pm 6,13$, sendo 6 do gênero masculino e 3 feminino. Destes, um foi classificado no estágio 1,5 da escala Hoehn e Yahr, dois classificados no estágio 2, quatro classificados no estágio 2,5 e dois classificados no estágio 3, com média geral de 2,38 $\pm 0,48$. A pontuação média na escala do MEEM foi de $26 \pm 3,64$. Quando ao tempo de diagnóstico a média foi de 7,6 $\pm 4,5$ anos. Apenas um indivíduo apresentava diagnóstico de hipertensão arterial. Todos os pacientes faziam uso de droga precursora de dopamina, três de dopamina endógena, três de agonista dopaminérgico e um de droga anticolinérgica. Tiveram 3 perdas no estudo: uma por falecimento e dois por mais de três faltas nas terapias.

Houve diferença estatisticamente significante na progressão da doença entre a avaliação pré e pós-tratamento apontada pela escala UPDRS na pontuação total $(p=0,03)$ e no domínio exame motor $(p=0,03)$, porém não houve diferença estatisticamente significante no domínio atividades de vida diária $(\mathrm{p}=0,93)$. Os dados podem ser observados na Tabela 1. 
Tabela 1 - Comparação da progressão da doença pela escala UPDRS pré e pós-intervenção

\begin{tabular}{c|c|c|c}
\hline & Pré & Pós & P \\
\hline UPDRS - AVD (score) & $9,88 \pm 4,67$ & $10,00 \pm 5,09$ & 0,93 \\
\hline UPDRS - Motor (score) & $24,55 \pm 10,86$ & $20,11 \pm 10,04$ & $0,03^{*}$ \\
\hline UPDRS - Total (score) & $34,44 \pm 14,48$ & $30,11 \pm 13,47$ & $0,03^{*}$ \\
\hline
\end{tabular}

UPDRS = Unified Parkinson Disease Rating Scale; AVD = Atividade de Vida Diária; score = pontuação. *diferença estatisticamente significante.

Na Tabela 2 estão registrados os valores referentes à avaliação do Best Test. Observam-se valores estatisticamente significantes para as seções II (limites de estabilidade) e IV (respostas posturais reativas) e para o escore total.

Score = pontuação. *diferença estatisticamente significante.

Tabela 2 - Valores referentes à avaliação do BESTest.

\begin{tabular}{c|c|c|c}
\hline & Pré & Pós & $\mathbf{P}$ \\
\hline $\begin{array}{c}\text { Seção I - Restrições } \\
\text { biomecânicas (score) }\end{array}$ & $11,44 \pm 3$ & $12,33 \pm 2,82$ & 0,16 \\
\hline $\begin{array}{c}\text { Seção II - Limites de } \\
\text { estabilidade (score) }\end{array}$ & $16,66 \pm 1,32$ & $17,77 \pm 1,3$ & $0,02^{*}$ \\
\hline $\begin{array}{c}\text { Seção III - Transições } \\
\text { (score) }\end{array}$ & $15,77 \pm 1,98$ & $16,11 \pm 1,83$ & 0,48 \\
\hline $\begin{array}{c}\text { Seção IV - Respostas } \\
\text { posturais reativas (score) }\end{array}$ & $15,88 \pm 1,76$ & $17 \pm 1,11$ & $0,04^{*}$ \\
\hline $\begin{array}{c}\text { Seção V - Orientação } \\
\text { sensorial (score) }\end{array}$ & $15[14-15]$ & $15[15-15]$ & 0,08 \\
\hline $\begin{array}{c}\text { Seção VI - Estabilidade } \\
\text { na marcha (score) }\end{array}$ & $17,22 \pm 2,94$ & $18,44 \pm 1,5$ & 0,23 \\
\hline Total (score) & $91,55 \pm 7,89$ & $96,55 \pm 6,46$ & $0,01^{*}$ \\
\hline
\end{tabular}

Quanto aos valores referentes à aplicação do protocolo de avaliação do balance, para o desfecho equilíbrio, os valores referentes à posição de Romberg com os olhos abertos e fechados, Tandem bilateral com os olhos abertos e fechados sobre superfície estável foram propositalmente omitidas da Tabela 3 porque foram constantes em seus valores iniciais e finais. Nas demais posições não houve diferença estatisticamente significante, como observado na tabela 3.

Tabela 3 - Diferenças pré e pós-intervenção quanto à análise de equilíbrio pelo protocolo do balance, sobre superfície estável: mediana [intervalo interquartílico].

\begin{tabular}{c|c|c|c}
\hline Testes & Pré & Pós & P \\
\hline Unipodal OA pé D (s) & $30[5,5-30]$ & $19,5[7,9-30]$ & 0,9 \\
\hline Unipodal OA pé E (s) & $30[5,1-30]$ & $24[13,3-30]$ & 0,9 \\
\hline Unipodal OF pé D (s) & $3[2-7,8]$ & $5,4[1,7-15,5]$ & 0,4 \\
\hline Unipodal OF pé E (s) & $3,7[1-12,7]$ & $6,7[5,6-12]$ & 0,1 \\
\hline
\end{tabular}

$\mathrm{OA}=$ olhos abertos; OF = olhos fechados; $\mathrm{D}$ = direito; $\mathrm{E}$ = esquerdo; valores em segundos (s).

Os valores referentes à posição de Romberg com os olhos abertos e fechados e à posição de Tandem bilateral com os olhos abertos foram propositalmente omitidas da Tabela 4 porque foram constantes em seus valores iniciais e finais. Houve diferença estatisticamente significante na posição de Tandem bilateral com os olhos fechados, sobre superfície instável. Os demais testes não apresentaram diferença estatisticamente significante, como apresentados na tabela 4. 
Tabela 4 - Diferenças pré e pós-intervenção quanto à análise de equilíbrio pelo protocolo do balance, sobre superfície instável: mediana [intervalo interquartílico].

\begin{tabular}{c|c|c|c}
\hline Testes & Pré & Pós & $\mathbf{P}$ \\
\hline Tandem OF pé D $(\mathrm{s})$ & $7[4,2-27,6]$ & $30[30-30]$ & $0,01^{*}$ \\
\hline Tandem OF pé E $(\mathrm{s})$ & $15[8-25]$ & $30[30-30]$ & $0,01^{*}$ \\
\hline Unipodal OA pé D (s) & $9,1[3,1-30]$ & $10,7[4,4-30]$ & 0,7 \\
\hline Unipodal OA pé E (s) & $14,7[6-30]$ & $12[8,3-30]$ & 0,9 \\
\hline Unipodal OF pé D (s) & $2[0,3-3,5]$ & $3[1,5-3,8]$ & 0,1 \\
\hline Unipodal OF pé E (s) & $4[1,7-5,7]$ & $4[2,6-6,8]$ & 0,3 \\
\hline
\end{tabular}

$\mathrm{OF}=$ olhos fechados; $\mathrm{OA}=$ olhos abertos; $\mathrm{D}=$ direito; $\mathrm{E}=$ esquerdo; valores em segundos (s); ${ }^{*}$ diferença estatisticamente significante.

\section{Discussão}

Considera-se as alterações do equilíbrio uma das principais características presente nos pacientes com DP, sendo objeto de estudo muito buscado nos últimos anos. A degeneração dos núcleos da base gera um padrão inibitório exacerbado, fazendo com que o paciente encontre dificuldade em modular as estratégias de equilíbrio, apresentando inadequada interação entre os sistemas responsáveis pelo equilíbrio corporal, como o sistemas vestibular, o visual e o proprioceptivo. Em consequência desta alteração, esses pacientes tendem a deslocar seu centro de gravidade para frente, dificultando a realização de movimentos compensatórios para readquirir a estabilidade, gerando, desta forma, frequente situações de quedas ${ }^{22}$, o que atesta a importância e necessidade de estudos sobre o tema equilíbrio na DP.

A maioria dos indivíduos que são diagnosticados com DP não procura um fisioterapeuta, até que os sinais e sintomas apareçam de forma clara. No entanto, é possível que um programa específico de exercícios, centrado nos principais déficits que são inevitáveis com a progressão da doença, possa ajudar os pacientes que ainda não apresentaram alterações. Aspectos como mobilidade e instabilidade postural, entre eles os distúrbios de equilíbrio, não respondem à terapia medicamentosa e cirúrgica, tornando a prevenção uma opção relevante para esses indivíduos ${ }^{10}$. Estudos tem investigado a eficácia de programas de exercício nos sintomas de pacientes com DP. A maioria desses trabalhos mostra que os pacientes podem beneficiar-se de programas de reabilitação, além do tratamento médico, e que quando esses programas estão sob supervisão fisioterapêutica são mais efetivos na melhora das tarefas da vida diária e das atividades motoras, mentais e emocionais, quando comparados a tratamentos não supervisionados ${ }^{14,15,23,24}$.

$O$ primeiro ensaio de tratamento fisioterápico na DP foi realizado por Gibberd et a ${ }^{25} \mathrm{em} 1981$. Vinte e três pacientes foram submetidos à facilitação neuromuscular proprioceptiva e ao método Bobath, visando melhorar o equilíbrio, festinação, rigidez e a mobilidade em geral. Após três meses foram reavaliados e submetidos por mais três meses à terapia concluindo, os autores, que não houve melhora com o tratamento realizado. Porém, há que se considerar que não foram utilizadas escalas conhecidas nas avaliações e não foi realizado estudo randomizado, pois na época da execução de tal estudo não haviam diretrizes robustas para pesquisas científicas como na atualidade.

Em recente revisão sistemática e metanálise de Tomlinson et a ${ }^{1 / 3}$, no ano de 2012, com inclusão de 29 ensaios clínicos sobre a intervenção da fisioterapia na DP, foi verificada melhora a favor da intervenção fisioterápica nos seguintes desfechos: velocidade da marcha metros por segundo; agilidade; mobilidade funcional (teste do alcance); equilíbrio por meio da escala de Berg e nos escores da UPDRS com diminuição da pontuação do domínio motor (média de 5,01 pontos) e do domínio atividades de vida (média de 1,36 pontos). As principais abordagens utilizadas pelos autores incluídos na pesquisa muito se assemelham ao protocolo desenvolvido e aplicado no presente estudo, como segue: terapia baseada no conceito Bobath, treino de equilíbrio e marcha com pistas visuais, sensoriais e auditivas e facilitação neuromuscular, entre outras ${ }^{13}$. Vale ressaltar que os resultados positivos descritos acima referentes à escala UPDRS e equilíbrio encontrados no estudo também corroboram com nossos achados.

A dificuldade em realizar duplas tarefas somadas à dificuldade de controlar de forma automática os movimentos, justificam as condutas mais utilizadas na fisioterapia - 0 treino com pistas externas e estratégias atencionais para controlar os movimentos, principalmente a marcha, à medida que aloca a atenção para o movimento, limitando a atenção disponível para outras tarefas. Tendo isso em vista, Souza ${ }^{26}$ verificou os efeitos de um treinamento motor em associação com tarefas 
cognitivas (visual e auditivo), de diferentes complexidades, sobre o desempenho em uma tarefa motora e na habilidade em gerenciar/dividir a atenção em pacientes com DP. Como conclusão observaram que o treino de dupla tarefa foi efetivo, com significância estatística, para a melhora da agilidade de forma isolada (média de diminuição de 2 segundos) e sob condição de dupla tarefa (média de 2,8 segundos), além da melhora nas deficiências cognitivas para as tarefas visuais (com diminuição média de porcentagem de erros por minuto de 17,38) e para as tarefas auditivas (com diminuição média de porcentagem de erros por minuto de 23,95) de indivíduos com DP. Mesmo que este estudo não aborde o desfecho equilíbrio e dificulte a comparação com nossos resultados, note-se a similaridade da intervenção entre ambos.

Na mesma linha, Yogev-Selimann et a ${ }^{27}$ realizaram estudo com o objetivo de analisar os efeitos da priorização da marcha ou da tarefa cognitiva sobre a velocidade da marcha em 20 pacientes com DP e 20 indivíduos saudáveis. Os indivíduos de ambos os grupos diminuíram de forma estatisticamente significante a velocidade da marcha (média de 0,21 $\mathrm{m} / \mathrm{s}$ ) enquanto realizavam a dupla tarefa. Adicionalmente, houve prejuízo significante no tempo do passo para o grupo DP (média de 0,08 s) e para indivíduos saudáveis (média de 0,05 s) e no tempo de oscilação para o grupo DP (média de $0,78 \%$ ) e para indivíduos saudáveis (média de $0,48 \%$ ). Este efeito negativo foi mais evidente no grupo DP.

Pelo exposto, fica claro que a maioria dos estudos que envolve o treinamento com duplas tarefas vem sendo utilizado para verificar a efetividade dessa intervenção na melhora da marcha. Esse fato despertou o interesse da pesquisa atual em verificar também a efetividade desse treinamento na melhora do equilíbrio.

Kwakkel et a ${ }^{28}$ fizeram levantamento das publicações sobre reabilitação na DP e identificaram seis revisões e 23 ensaios clínicos controlados envolvendo 1063 pacientes. Concluíram que houve melhora na postura, equilíbrio e marcha, porém os sintomas que haviam sido treinados mais intensamente e de maneira específica foram os que mais obtiveram bons resultados, o que indica que terapias específicas são essenciais para esses indivíduos, como demonstrado no estudo de Christofoletti et $a^{29}$, que analisaram em um ensaio clínico controlado, a eficácia de um programa de treinamento fisioterapêutico sobre o equilíbrio estático e dinâmico em pacientes com DP idiopática. Os indivíduos foram avaliados e submetidos a treinamento composto por exercícios fisioterapêuticos que estimulassem o equilíbrio por meio de atividades específicas para este desfecho. O grupo experimental apresentou melhora significativa do equilíbrio em relação ao controle, sendo a intervenção fisioterápica específica ao promover importante melhoria no equilíbrio desses sujeitos, o que reforça a necessidade de intervenções específicas para esses indivíduos.

Em contraste, Ashburn et a ${ }^{\beta 0}$, também em ensaio clínico randomizado avaliaram a eficácia de um programa de exercícios fisioterapêuticos baseado em atividades de fortalecimento muscular, alongamentos, treino de equilíbrio, marcha e atividades cognitivas na melhora do equilíbrio, agilidade, qualidade de vida e na diminuição dos eventos de quedas em pacientes com DP. Participaram do estudo 141 pacientes, divididos em grupo intervenção e grupo controle, avaliados previamente à intervenção, ao final do período de oito semanas de intervenção e após seis meses de follow up, não havendo melhora na agilidade e no equilíbrio após o protocolo desenvolvido. Note que a intervenção pouco singular para o treino de equilíbrio não foi capaz de gerar impacto positivo nos valores desse desfecho para o grupo estudado.

Em um estudo que compara o BESTest em sua versão reduzida com a Escala de Equilíbrio de Berg, os resultados apontam que o BESTest se mostra mais sensível na avaliação de pacientes com DP moderada e com déficits sutis de equilíbrio, quando comparado à escala de Berg, pois muitos pacientes que tem déficits notáveis de equilíbrio alcançavam pontuações máximas na escala de Berg impossibilitando uma avaliação adequada ${ }^{31}$. Este fato justifica a escolha dos autores pelo BESTest. Conradsson et $a^{\beta 2}$ investigou os efeitos de um treinamento específico de equilíbrio, baseado em atividades em posturas instáveis e treino de dupla tarefa, durante 12 semanas, em 5 indivíduos com DP e classificados nos estágios de leve a moderado, semelhante ao atual estudo. Para avaliação do equilíbrio foi utilizado o BESTest em sua versão reduzida, e foi encontrado melhora nas pontuações do teste em 4 participantes. Os autores discutem que apesar do número amostral reduzido, estudos anteriores apontam que uma melhora maior que 3,5 pontos individualmente e maior que 1 ponto na escala quando considerado o grupo, indica uma mudança real no equilíbrio. Sugerem portanto, que treinamentos específicos para o equilíbrio seja uma ferramenta promissora para reabilitação.

Em síntese, um crescente corpo de evidências indica que o protocolo de treinamento com duplas tarefas utilizado no presente estudo pode ser eficaz para o tratamento dos sinais motores e equilíbrio da DP, entretanto há poucas evidências sobre a melhora do equilíbrio após esse tipo de abordagem.

Como limitação do estudo há que se considerar o tamanho amostral reduzido, que deve-se, em parte, à própria incidência da doença de Parkinson, mas que pôde levar-nos a incorrer ao erro tipo II. Ensaios clínicos aleatórios também são necessários com grupo controle, bem como estudos com seguimento (follow up), por serem ferramentas mais poderosas para obtenção de evidências em pesquisas em saúde. 


\section{Considerações Finais}

Os resultados obtidos apontam melhoras nos resultados estudados referentes ao equilíbrio e escores motor e total da UPDRS, o que sugere ser um tratamento adequado no grupo de indivíduos estudado. Finalmente, espera-se contribuir com futuras pesquisas que relacionem o equilíbrio de pacientes com DP com a fisioterapia.

\section{Referências Bibliográficas}

1. Horak FB, Macpherson JM. Postural orientation and equilibrium. In: Rowel LB, Shepherd JT, editors. Handbook of Phisiology: Section 12, Exercise Regulation and Integration of Multiple Systems. New York: Oxford University Press. 1996;225-92.

2. Horak FB. Postural orientation and equilibrium: what do we need to know about neural control of balance to prevent falls? Rev Age and Ageing. 2006;35-S2.

3. MaiaAC, Rodrigues-de-Paula F, Magalhães LC, Teixeira RLL. Cross-cultural adaptation and analysis of the psychometric properties of the Balance Evaluation Systems Test and MiniBESTest in the elderly and individuals with Parkinson's disease: application of the Rash model. Braz J Phys Ther. 2013;17(3):195-217.

4. Navarro-Peternella FM, Marcon SS. Quality of life of a person with Parkinson's disease and the relationship between the time of evolution and the severity of the disease. Rev Lat Am Enf. 2012;20(2).

5. Gonçalves GB, Leite MAA, Pereira JS. Influência das distintas modalidades de reabilitação sobre as disfunções motoras decorrentes da Doença de Parkinson. Rev Bras Neurol. 2011;47(2):22-30.

6. Scalzo PL, Flores CR, Marques JR, Robini SCO, Teixeira AL. Impact of changes in balance and walking capacity on the quality of life in patients with Parkinson's disease. Arq Neuropsiquiatr. 2012;70(2):119-124.

7. Duncan RP, Leddy ALL, Earhart GM. Five tives sit-to-stand test performance in Parkinson's disease. Arch Phys Med Rehabil. 2011;92.

8. Takeuti T, Maki T, Silva CVR, Soares AJ, Duarte J. Correlação entre equilíbrio e incidência de quedas em pacientes portadores de doença de Parkinson. Rev Neurocienc. 2011;19(2):237-243.

9. Jacobs JV, Dimitrova DM, Nutt JG, Horak FB. Can stooped posture explain multidirectional postural instability in patients with Parkison's disease? Exp Brain Res. 2005;166(1):77-88.

10. Horak FB, Wrisley DM, Frank J. The Balance Evaluation Systems Test (BESTest) to Differentiate Balance Deficits. Phys Ther. 2009;89(5):484-498.

11. Teixeira NB, Alouche SR. O Desempenho da Dupla Tarefa na Doença de Parkinson. Rev Bras Fisiot. 2007;11(2):127-132. 12. Yogev-Seligmann G, Giladi N, Brozgol M, Hausdorff JM. A training program to improve gait while dual tasking in patients with Parkinson's disease: A pilot study. Arch Phys Med Rehabil. 2012;93.

13. Tomlinson CL, Patel S, Meek C, Herd CP, Clarke CE, Stowe R, et al. Physiotherapy intervention in Parkinson's disease: systematic review and meta-analysis. BMJ. 2012;345:e5004.

14. Wong-Yu ISK, Mak MKY. Multi-dimensional balance training programme improves balance and gait performance in people with Parkinson's disease: A pragmatic randomized controlled trial with 12-month follow-up. Parkinsonism and Related Disorders, 2015.

15. Muñoz-Hellín E, Cano-de-la-Cuerda R, Miangolarra-Page JC. Visual cues as a therapeutic tool in Parkinson's disease. A systematic review. Rev Esp Geriatr Gerontol 2013; 48(4):190-7.

16. Fok $P$, Farrell M, McMeeken J. The effect of dividing attention between walking and auxiliary tasks in people with Parkinson's disease. Hum MovSci. 2012;31(1):236-46.

17. Brauer SG, Woollacott MH, Lamont R, Clewett, S, O'Sullivan,J, Silburn P et al. Single and dual task gait training in people with Parkinson's disease: a protocol for a randomized controlled trial. BMC Neurol. 2011; 11:90.

18. Hoehn MM, Yahr MD. Parkinsonism: onset, progression and mortality. Neurol. 1967;17:427-42.

19. Folstein MF, Folstein SE, Mchugh PR. MiniMental State: a practical method for grading the cognitive state of patients for clinician. Journal of Psychiatric Research, v. 12, p. 189-198, 1975.

20. Fahn S, Elton RL, Members of the UPDRS Development Committee. In: Fahn S, Marsden CD, Calne DB, Goldstein M. eds. Recent developments in Parkinsons disease, Vol 2. Florham Park, NJ. Macmillan Healthcare Information 1987, 153-163, $293-304$. 21. Maia AC. Tradução e adaptação para o português - Brasil do Balance Evaluation Systems Test e do MiniBESTTest e análise de suas propriedades psicométricas em idosos e indivíduos com doença de Parkinson. [dissertação] Belo Horizonte: Escola de Educação Física, Fisioterapia e Terapia Ocupacional da Universidade Federal de Minas Gerais, 2012. 
22. Flores FT, Rossi AG, Schmidt PS. Avaliação do equilíbrio corporal na doença de Parkinson. Arq Int Otorrinolaringol. 2011;15(2):142-150.

23. Loureiro CMV. Efeitos da musicoterapia na qualidade de vida visual de portadores de neurite óptica desmielinizante [tese]. Belo Horizonte (MG): Universidade Federal de Minas Gerais; 2009.

24. Dereli EE, Yaliman A. Comparison of the effects of a physiotherapist supervised exercise programme and a selfsupervised exercise programme on quality of life in patients with Parkinson's disease. Clin Rehabil. 2010;24(4):352-62.

25. Gibbers FB, Page NGR, Spencer KM, Kinnear EML, Hawksworth JB. Controlled trial of physiotherapy and occupational therapy for Parkinson's disease. Br Med J. 1981;282:1196.

26. Souza, CB. Efeitos de um treinamento em condições de dupla-tarefa sobre o desempenho motor e habilidade de dividir a atenção em pacientes com doença de Parkinson [dissertação]. São Paulo (SP): Universidade de São Paulo; 2008.

27. Yogev-Seligmann G, Rotem-Galili Y, Dickstein R, Giladi N, Hausdorff JM. Effects of explicit prioritization on dual task walking in patients with Parkinson's disease. J Gait \& Postur. 2012;35(4):641-646.

28. Kwakkel G, De Goede CJT, Vab Wegen EEH. Impact of physical therapy for Parkinson's disease: a critical review of the literature. Parkin Relat Disord. 2007;13:478-87.

29. Christofoletti G, Freitas RT, Cândido ER, Cardoso CS. Eficácia de tratamento fisioterapêutico no equilíbrio estático e dinâmico de pacientes com doença de Parkinson. Fisioter Pesq. 2010;17(3):259-63.

30. Ashburn A, Fazakarley L, Ballinger C, Pickering R, McLellan LD, Fitton C. A randomised controlled trial of a home based exercise programme to reduce the risk of falling among people with Parkinson's disease. J Neurol Neurosurg Psychiatry. 2007;78:678-684.

31.King LA, Priest KC, Salarian A, Pierce D, Horak FB. Comparing the Mini-BESTest with the Berg Balance Scale to Evaluate Balance Disorders in Parkinson's Disease. Parkinson's Disease, 2012.

32. Conradsson D, Löfgren N, Stahle A, Franzén E. Is Highly Challenging and Progressive Balance Training Feasible in

Older Adults With Pakinson's Disease? Arch Phys Med Rehab. 2014; 95(5):1000-1003.

\section{Isabela Andrelino de Almeida}

Endereço para correspondência - Rua: Marcílio Dias, n²73, apto 403, Bairro: Caiçaras, CEP: 86015-620, Londrina, PR, Brasil

E-mail: isabelaandrelino@hotmail.com

Lattes: http://lattes.cnpq.br/5917675408140885

Maria Eduarda Brandão Bueno - dudaa_brandao@hotmail.com

Ana Carolina dos Reis Andrello - ana_andrello@hotmail.com

Cyntia Letícia Batistetti - cyntia_lb@hotmail.com

Luana Beatriz Lemes - luana_beatrizlemes@hotmail.com

Natália Mariano Barboza - nat_barboza@hotmail.com

Lucio Baena de Melo - lucio@luciobaena.com

Suhaila Mahmoud Smaili Santos - suhaila@uel.br

Enviado em 18 de maio de 2014. Aceito em 18 de julho de 2015. 
